\section{Tendência de bullying verbal, violência doméstica e envolvimento em brigas com armas entre adolescentes das capitais brasileiras de 2009 a 2015}

\author{
Trends in verbal bullying, domestic violence, \\ and involvement in fights with firearms among \\ adolescents in Brazilian state capitals \\ from 2009 to 2015
}

\section{Tendencia de bullying verbal, violencia doméstica e implicación en peleas con armas entre adolescentes de las capitales brasileñas de 2009 a 2015}

Aline Natália Silva 1

Emanuele Souza Marques 2

Maria Fernanda Tourinho Peres 3

Catarina Machado Azeredo 1

\section{Resumo}

Objetivou-se analisar a tendência temporal de bullying verbal, a violência doméstica e o envolvimento em brigas com armas entre adolescentes, nas capitais brasileiras, entre 2009 e 2015. Fez-se estudo de tendência, com uso de dados da Pesquisa Nacional de Saúde do Escolar (PeNSE) realizada em 2009, 2012 e 2015, com escolares do 9o ano do Ensino Fundamental de escolas públicas e privadas nas 26 capitais do país e no Distrito Federal. Analisou-se a ocorrência de violência doméstica e de bullying verbal, bem como o envolvimento em brigas com uso de armas branca e de fogo nos 30 dias anteriores à entrevista. Realizou-se regressão logística ajustada para características sociodemográficas e comportamentais, com análise de cada tipo de violência no Brasil e nas capitais, segundo sexo. As tendências foram espacializadas. Para o conjunto das capitais, houve aumento, entre 2009 e 2015, de 12\% (IC95\%: 1,11-1,14) para violência doméstica, de 10\% (IC95\%: 1,08-1,11) para bullying verbal, de 7\% (IC95\%: 1,05-1,09) para envolvimento em brigas com armas de fogo e de 7\% (IC95\%: 1,05-1,08) para envolvimento em brigas com arma branca. Em todas as capitais, houve aumento na violência doméstica. Em 96,3\%, 70,4\% e 62,9\% dessas cidades, observou-se aumento de bullying verbal, envolvimento em briga com arma branca envolvimento em briga com arma de fogo, respectivamente. Nas demais capitais, observou-se tendência estacionária. Identificou-se tendência de aumento das violências na maioria das capitais, evidenciando a necessidade de implementação de políticas públicas que contribuam para minimizar esse problema entre os adolescentes.

Estudos de Séries Temporais; Violência; Adolescente
Correspondência

C. M. Azeredo

Universidade Federal de Uberlândia.

Av. Pará 1720, Bloco 2U, Uberlândia. MG 38400-902, Brasil. catarina.azeredo@ufu.br

1 Universidade Federal de Uberlândia, Uberlândia, Brasil. 2 Universidade do Estado do Rio de Janeiro, Rio de Janeiro, Brasil.

3 Faculdade de Medicina, Universidade de São Paulo, São Paulo, Brasil. 


\section{Introdução}

A violência é a quarta principal causa de mortes em pessoas de 15 a 44 anos no mundo 1. Em países da América Latina e Caribe, a taxa de homicídios entre adolescentes é cinco vezes maior que a média mundial 2. No Brasil, no ano de 2016, cerca de 49,1\% dos óbitos entre jovens de 15 a 19 anos foram em decorrência de homicídios, que representam a principal causa de mortalidade nessa faixa etária ${ }^{3}$. Internacionalmente, o Brasil aparece entre os dez países com maiores taxas de mortalidade por homicídios entre jovens de 15 a 19 anos 2. Por sua vez, o excesso de lesões, originado da ocorrência de episódios de violência, é o principal problema de saúde entre pessoas com idade de 10 a 24 anos 4 . Dentre as consequências da violência interpessoal, observam-se internações decorrentes de ferimentos físicos, piora na qualidade de vida, transtorno de estresse pós-traumático, depressão, ideação suicida e mortes $5,6,7,8$.

Quanto aos tipos de violência entre adolescentes, o bullying verbal 2,9 e a violência doméstica 10 são as mais prevalentes. Embora acometa aproximadamente 10\% dos adolescentes brasileiros, o envolvimento em brigas com utilização de armas de fogo e arma branca merece destaque, por estar associado aos ferimentos mais graves e fatais 6,11.

Estudos sobre a tendência de violência entre jovens mostram evoluções diferentes entre os países. Nos Estados Unidos, houve tendência de redução no envolvimento em luta física entre adolescentes no período de 2002 a 2014 12, além de redução de bullying e de maus-tratos infantis nos anos de 2003 a 2011 13, após a implementação de ações no ambiente familiar e escolar para a minimização de violência 13,14. Em contrapartida, entre jovens canadenses, houve tendência crescente de agressão física entre os anos de 1993 e 2010 15. No Brasil, em Belo Horizonte e região metropolitana (Minas Gerais), evidenciou-se aumento de homicídios em quase todas as faixas etárias, especialmente entre os homens 16. Em relação à tendência de homicídios entre adultos brasileiros, encontraram-se nove trajetórias distintas entre os anos de 1991 e 2010, sendo mais frequente a trajetória de baixo crescimento, com aumento de cerca de $56 \%$ na taxa de mortalidade por homicídios (28,1\% dos municípios analisados), seguida da trajetória de estabilização, com aproximadamente 3\% de aumento nas mortes por homicídios (26,52\% dos municípios estudados) 17.

Embora estudos sobre tendência de violência entre adolescentes brasileiros tenham sido realizados recentemente, a abordagem do bullying foi somente descritiva 18 , sem presença de testes que possibilitem a análise de tendência temporal desse tipo de violência. No tocante à avaliação da evolução da violência doméstica e da violência com uso de armas de fogo e branca ${ }^{19}$, as análises realizadas não foram ajustadas de maneira a incluir variáveis de confusão importantes no modelo, como uso de álcool, cigarro e outras drogas 8,12 .

Diante do exposto, estudos de tendência de violência são necessários para a identificação de grupos mais vulneráveis, análise da evolução da violência no decorrer do tempo, além do acompanhamento da eficácia de políticas adotadas para a minimização desse fenômeno ${ }^{9}$. Além disso, como uma forma de violência muitas vezes aumenta o risco de envolvimento em outras formas, a avaliação da tendência de diferentes tipos de violência permite uma compreensão mais abrangente da vulnerabilidade dos adolescentes brasileiros. Nesse sentido, o objetivo do presente trabalho é analisar a tendência temporal de vitimização por bullying verbal, violência doméstica e envolvimento em brigas com uso de armas entre adolescentes escolares no período de 2009 a 2015, residentes nas capitais brasileiras e no Distrito Federal.

\section{Métodos}

O presente estudo utilizou os dados das três edições da Pesquisa Nacional de Saúde do Escolar (PeNSE), realizadas nos anos de 2009, 2012 e 2015, com estudantes do 9o ano do Ensino Fundamental. A PeNSE é um inquérito transversal realizado pelo Instituto Brasileiro de Geografia e Estatística (IBGE), em parceria com os Ministérios da Educação e da Saúde, com questões referentes aos fatores de risco e proteção para a saúde dos adolescentes escolares brasileiros 20,21,22.

A amostragem da PeNSE foi realizada em conglomerados, em múltiplos estágios, nas três edições da pesquisa. Foram utilizados somente os dados coletados para as capitais dos estados brasileiros e o 
Distrito Federal, para permitir a comparabilidade entre os três anos da pesquisa. Para a seleção das escolas que participariam da pesquisa em cada um dos três anos, foram utilizados dados do Censo Escolar dos anos de 2007, 2010 e 2013, respectivamente, e as instituições de ensino que possuíam turmas do 9 o ano do Ensino Fundamental foram incluídas na amostra. As escolas com uma ou duas turmas do $9 \underline{\text { o }}$ ano do Ensino Fundamental tiveram uma turma selecionada, e aquelas com três ou mais turmas tiveram duas turmas selecionadas. Também foi garantida a proporcionalidade entre as instituições de ensino públicas e privadas, conforme a quantidade de escolas com turmas de $9 \underline{o}$ ano cadastradas no Censo Escolar 20,21,22.

Os dados foram coletados com a utilização de microcomputadores de mão personal digital assistant (PDA) na PeNSE 2009 e de smartphones nas edições de 2012 e 2015. Os questionários foram preenchidos pelos escolares em todas as edições, sem necessidade de interferência do entrevistador 20,21,22. A participação nas três edições da PeNSE foi voluntária, e não houve identificação dos escolares nem das escolas 20,21,22.

Na PeNSE foram aferidas informações referentes aos seguintes aspectos: condições socioeconômicas; contextos familiares; hábitos alimentares; realização de atividade física; uso de cigarro, álcool e outras drogas; atividade sexual; violência, segurança e acidentes; hábitos de higiene; saúde mental; uso de serviços de saúde; dentre outros. Demais informações metodológicas estão descritas em Oliveira et al. 23 .

Os inquéritos utilizados (PeNSE 2009, PeNSE 2012 e PeNSE 2015) foram aprovados pela Comissão Nacional de Ética e Pesquisa (Conep), sob os respectivos registros: no 11.537, no 16.805 e no 1.006 .467 .

\section{Variáveis de interesse}

\section{- Violência}

A vitimização por bullying verbal foi identificada a partir da pergunta "Nos últimos 30 dias, com que frequência algum de seus colegas de escola te esculacharam, zoaram, mangaram, intimidaram ou caçoaram tanto que você ficou magoado/incomodado/aborrecido/ofendido/humilhado?”. Foram considerados vítimas os escolares que responderam "às vezes", "na maior parte das vezes" e "sempre". Aqueles que responderam "nenhuma vez" e "raramente" foram considerados não vítimas, uma vez que a repetição é uma das características do bullying 24. Para a violência doméstica, utilizou-se a pergunta "Nos últimos 30 dias, quantas vezes você foi agredido fisicamente por um adulto de sua família?". Os escolares que responderam "uma vez ou mais" foram classificados como vítimas desse tipo de violência. O envolvimento dos adolescentes em violência com uso de arma de fogo foi avaliado pela pergunta "Nos últimos 30 dias, você esteve envolvido(a) em alguma briga em que alguma pessoa usou arma de fogo, como revólver ou espingarda?”. O envolvimento em briga com arma branca seguiu a mesma estrutura, substituindo somente arma de fogo por "outra arma como faca, canivete, peixeira, pedra, pedaço de pau ou garrafa". As opções de resposta para as duas perguntas foram "sim” ou "não". Aqueles que responderam "sim" foram considerados envolvidos em briga com arma de fogo ou com arma branca, respectivamente.

\section{- Sociodemográficas e comportamentais}

Para a descrição da população de estudo e ajuste, foram utilizadas as seguintes variáveis: sexo (masculino e feminino), raça/cor (branca, preta, parda, amarela e indígena), idade (em anos), escolaridade materna (Ensino Fundamental incompleto, Ensino Fundamental completo, Ensino Médio completo e Ensino Superior completo) e dependência administrativa da escola (pública e privada). Além dessas, variáveis comportamentais como uso de álcool, de cigarro e de outras drogas, pelo menos uma vez nos últimos 30 dias (sim e não), foram incluídas para descrição e ajuste, pois foram utilizadas em outros estudos sobre violência 8,12 . 


\section{Análises de dados}

A variável escolaridade materna apresentou cerca de 18\%, 17\% e 23\% de dados faltantes (missing data), respectivamente nos anos de 2009, 2012 e 2015. Para lidar com esse elevado percentual de dados faltantes, realizou-se a imputação múltipla por equações em cadeias, que consiste em uma sequência de modelos de imputação e, para cada variável faltante, um modelo foi utilizado 25 . Também foram incluídas na imputação outras variáveis com quantidades menores de dados faltantes para se obter uma informação mais completa e precisa. A partir das informações obtidas com o banco imputado, foram realizadas as análises do presente trabalho.

Todas as análises foram realizadas considerando-se a ponderação para a estrutura amostral complexa, de modo a representar a população de escolares brasileiros residentes nas 26 capitais dos estados brasileiros mais o Distrito Federal.

Para verificar a tendência dos quatro tipos de violência entre os adolescentes para o período, foram realizadas análises de regressão logística, utilizando como variável dependente o envolvimento em cada um dos quatro tipos de violência. $\mathrm{O}$ ano de realização dos inquéritos foi considerado como variável independente. Os modelos foram ajustados para as variáveis sociodemográficas e comportamentais (uso de álcool, cigarro e outras drogas). As análises foram estratificadas por sexo e pelas 26 capitais dos estados brasileiros, além do Distrito Federal. Utilizou-se como ponto de corte valor de $\mathrm{p}$ $<0,05$ para estabelecer a significância estatística. Todas as análises foram realizadas no software Stata versão 12.1 (https://www.stata.com).

Os resultados de tendência para cada tipo de violência em cada capital foram espacializados, com o uso do software QGIS (https://qgis.org/en/site/) para edição de arquivo digital referente ao mapa base do Brasil, disponibilizado pelo IBGE.

\section{Resultados}

A amostra total foi composta de 173.310 escolares residentes nas 26 capitais brasileiras e no Distrito Federal. Houve predominância do sexo feminino na amostra, em todo período estudado. Os níveis de escolaridade materna mais relatados foram o Ensino Fundamental incompleto e o Médio completo. A maior parte dos adolescentes se autodeclarou branca (em 2009) e parda (2012 e 2015). Em relação à dependência administrativa da escola, mais de 70\% dos entrevistados estudavam em escolas públicas (Tabela 1).

Dentre os tipos de violência estudados, o bullying verbal e a violência doméstica tiveram as maiores prevalências em todo o período, seguidos da violência com uso de arma branca e de fogo (Tabela 1; Figuras 1a e 1b).

Observou-se aumento de 12\% (IC95\%: 1,11-1,14) para a violência doméstica, de 10\% (IC95\%: 1,08-1,11) para o bullying verbal, de 7\% (IC95\%: 1,05-1,09) no envolvimento em briga com uso de arma de fogo e de 7\% (IC95\%: 1,05-1,08) no envolvimento em brigas com arma branca, no período total, de 2009 a 2015 (Tabela Suplementar: http://cadernos.ensp.fiocruz.br/site/public_site/arquivo/ supl-e00195118_9820.pdf). As maiores diferenças nas prevalências entre os sexos foram observadas no envolvimento em briga com utilização de qualquer tipo de arma, sendo cerca de duas vezes mais prevalente no sexo masculino (Figuras 1c e 1d). Mesmo com a diminuição nas prevalências de violências com uso de armas de 2012 a 2015 (Figuras 1c e 1d), no período total (2009 a 2015), foram observadas tendências de aumento (Tabela Suplementar: http://cadernos.ensp.fiocruz.br/site/public_site/ arquivo/supl-e00195118_9820.pdf).

A Figura 2 mostra os mapas de tendência das violências em todas as capitais brasileiras, no período avaliado. Observa-se que tanto a vitimização por violência doméstica quanto por bullying verbal tiveram tendência de aumento significante em todas as capitais brasileiras, exceto João Pessoa (Paraíba), na qual a vitimização por bullying verbal apresentou tendência estacionária (Figuras 2a e 2b).

Quanto ao envolvimento dos adolescentes em briga com arma de fogo, observou-se tendência de aumento para 17 capitais e tendência estacionária para 10, com aumento mais acentuado em São Luís (Maranhão) e em Teresina (Piauí), ambas capitais da Região Nordeste (Figura 2d). Em relação ao envolvimento em briga com arma branca, ocorreu tendência de aumento em 19 capitais, sendo 


\section{Tabela 1}

Perfil sociodemográfico e prevalência de bullying verbal, violência doméstica e envolvimento em briga com arma branca e com arma de fogo entre adolescentes escolares brasileiros. Pesquisa Nacional de Saúde do Escolar (PeNSE) 2009, 2012 e 2015.

\begin{tabular}{|c|c|c|c|}
\hline \multirow[t]{2}{*}{ Características gerais da amostra } & $2009(n=60.937)$ & $2012(n=61.145)$ & $2015(n=51.192)$ \\
\hline & $\%(I C 95 \%)$ & $\%(I C 95 \%)$ & $\%(I C 95 \%)$ \\
\hline \multicolumn{4}{|l|}{ Sexo } \\
\hline Masculino & $47,5(46,8-48,1)$ & $49,2(48,4-49,9)$ & $49,2(48,3-50,0)$ \\
\hline Feminino & $52,5(51,9-53,2)$ & $50,8(50,1-51,6)$ & $50,8(49,9-51,7)$ \\
\hline \multicolumn{4}{|l|}{ Idade (anos) } \\
\hline$\leq 13$ & $24,4(23,3-25,5)$ & $19,0(18,1-19,9)$ & $20,3(19,3-21,4)$ \\
\hline 14 & $47,2(46,0-48,3)$ & $50,1(49,0-51,2)$ & $52,4(51,3-53,6)$ \\
\hline 15 & $18,2(17,2-19,3)$ & $19,4(18,5-20,2)$ & $18,5(17,5-19,4)$ \\
\hline$\geq 16$ & $10,2(09,4-10,9)$ & $11,5(10,6-12,4)$ & $8,8(8,1-9,4)$ \\
\hline \multicolumn{4}{|l|}{ Raça/Cor } \\
\hline Branca & $40,2(38,9-41,4)$ & $37,7(36,1-39,3)$ & $36,5(34,7-38,3)$ \\
\hline Preta & $12,7(12,1-13,4)$ & $14,2(13,4-14,9)$ & $13,5(12,7-14,3)$ \\
\hline Amarela & $3,7(3,4-4,0)$ & $4,5(4,2-4,8)$ & $5,0(4,7-5,4)$ \\
\hline Parda & $39,3(38,2-40,4)$ & $39,9(38,6-41,2)$ & $41,8(40,3-43,2)$ \\
\hline Indígena & $4,1(3,8-4,3)$ & $3,7(3,4-3,9)$ & $3,2(2,9-3,4)$ \\
\hline \multicolumn{4}{|l|}{ Escolaridade materna } \\
\hline Ensino Fundamental incompleto & $36,1(34,7-37,5)$ & $33,1(31,3-34,9)$ & $27,1(25,5-28,6)$ \\
\hline Ensino Fundamental completo & $14,6(14,1-15,1)$ & $15,7(15,1-16,3)$ & $13,8(13,1-14,5)$ \\
\hline Ensino Médio completo & $31,1(30,0-32,2)$ & $34,9(33,8-35,9)$ & $34,8(33,8-35,8)$ \\
\hline Ensino Superior completo & $18,2(17,0-19,4)$ & $16,3(14,5-18,1)$ & $24,3(22,2-26,5)$ \\
\hline \multicolumn{4}{|l|}{ Dependência administrativa da escola } \\
\hline Pública & $79,2(77,9-80,5)$ & $74,5(70,7-78,3)$ & $72,9(68,8-77,0)$ \\
\hline Privada & $20,8(19,5-22,1)$ & $25,5(21,7-29,3)$ & $27,1(23,0-31,2)$ \\
\hline Uso de álcool & $27,1(26,3-27,9)$ & $26,8(25,8-27,7)$ & $23,2(22,2-24,2)$ \\
\hline Uso de cigarro & $6,2(5,8-6,7)$ & $6,1(5,6-6,5)$ & $5,3(4,9-5,8)$ \\
\hline Uso de drogas & $3,3(3,0-3,6)$ & $3,8(3,4-4,2)$ & $2,4(2,2-2,7)$ \\
\hline Bullying verbal & $14,2(13,6-14,7)$ & $16,5(15,9-17,1)$ & $21,7(21,1-22,4)$ \\
\hline Violência doméstica & $9,5(9,0-10,0)$ & $11,6(11,1-12,1)$ & $16,2(15,5-16,8)$ \\
\hline Briga com uso de arma branca & $6,1(5,7-6,4)$ & $8,3(7,8-8,7)$ & $8,2(7,6-8,7)$ \\
\hline Briga com uso de arma de fogo & $4,0(3,7-4,3)$ & $6,9(6,6-7,3)$ & $5,6(5,2-6,0)$ \\
\hline
\end{tabular}

IC95\%: intervalo de 95\% de confiança.

estacionária nas demais (Figura 2c). Além disso, nas capitais da Região Sul, para as formas de violência mais graves, foram observadas tendências estacionárias (Figuras 2c e 2d).

\section{Discussão}

O presente estudo identificou tendência de aumento para todos os tipos de violência entre os escolares brasileiros para ambos os sexos, no período de 2009 a 2015. Tendência crescente de violência doméstica foi observada em todas as capitais brasileiras: bullying verbal em 96,3\%, envolvimento em briga com armas brancas em 70,4\% e com arma de fogo em 62,9\%.

A violência doméstica foi o tipo que apresentou aumento mais acentuado durante o período avaliado, passando de 9,5\% para 16,2\% entre escolares das capitais brasileiras. Mesmo sendo proibida por lei 26, a violência física como forma de educar os filhos ainda é recorrente em famílias brasileiras 27 , sendo socialmente aceita, desde que não cause lesão física visível nas vítimas 28 . Uma possível expli- 
Figura 1

Tendência de bullying verbal, violência doméstica e envolvimento em brigas com uso de arma branca e com arma de fogo entre escolares brasileiros, segundo sexo, para os anos de 2009, 2012 e 2015.

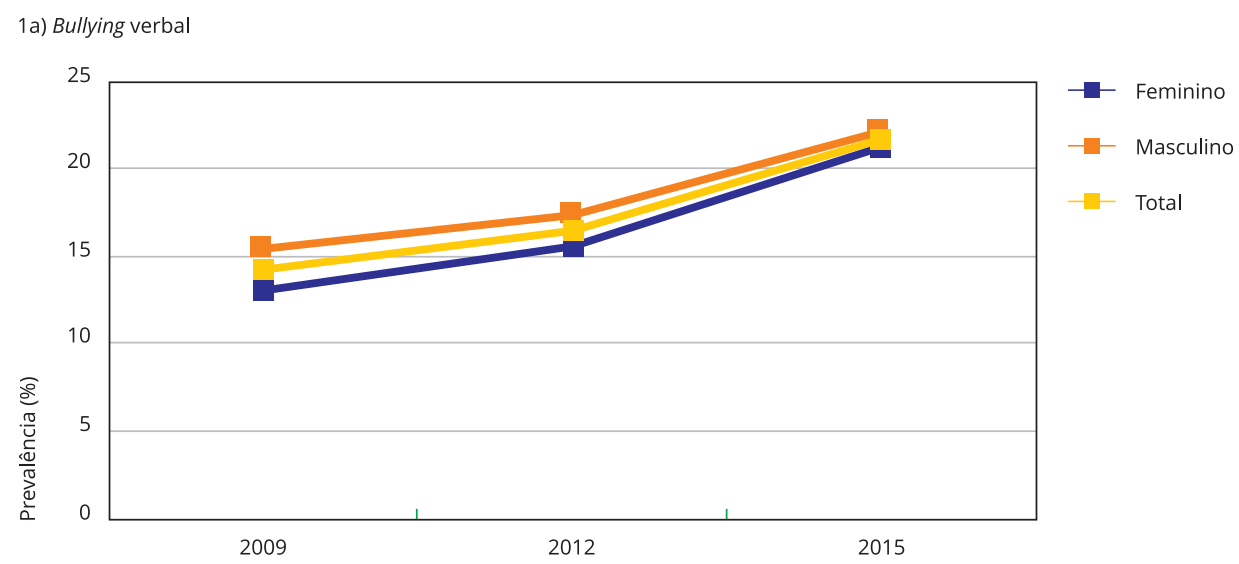

1b) Violência doméstica

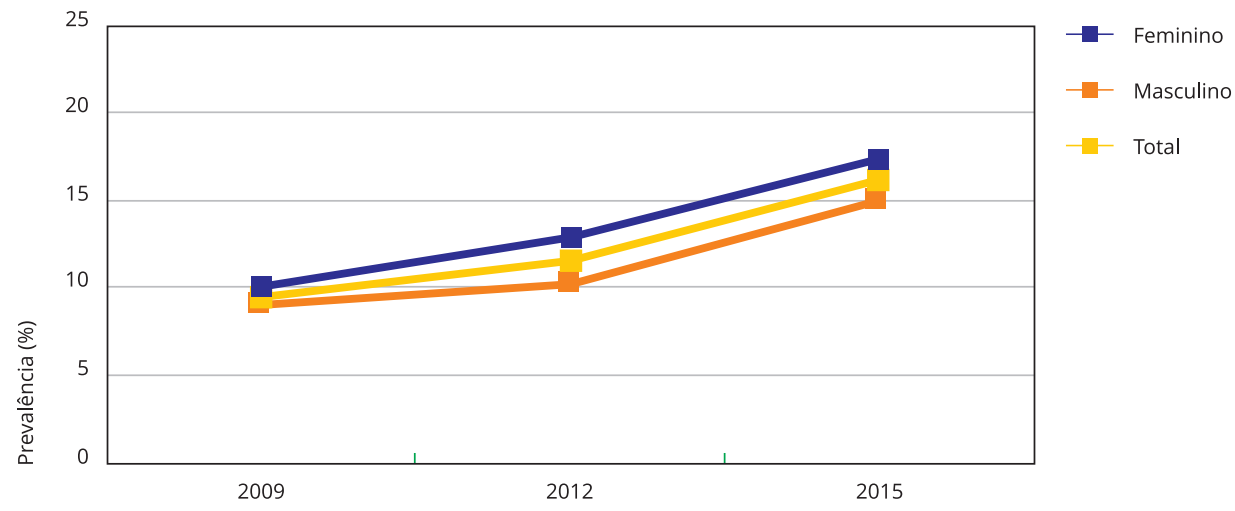

(continua)

cação para a tendência de aumento da violência doméstica entre escolares é o aumento da conscientização por parte dos adolescentes sobre esse tipo de violência, o que influencia o aumento do relato e não da violência em si. Além disso, pode estar ocorrendo a quebra do "pacto do silêncio" (situação na qual a vítima não relata a agressão sofrida por ter vínculo com o agressor e por sofrer ameaça ${ }^{29}$ ), e isso favoreceria o aumento nos relatos dos casos desse tipo de vitimização.

Ademais, é importante pontuar que este resultado está em desacordo com o observado em estudos feitos em países de alta renda, onde foi encontrada diminuição da violência perpetrada pelos pais no ambiente familiar 13,30. Em alguns países de alta renda, foram desenvolvidas políticas para minimização desse tipo de violência e também programas de parentagem, o que contribuiu para redução dos maus-tratos infantis e do comportamento agressivo entre crianças 30,31.

É importante ressaltar que a vivência de episódios de violência doméstica influencia a percepção em relação a esse comportamento, considerando-o normal e aceito. Dessa forma, os envolvidos passam a utilizar a violência como estratégia para resolução de conflitos, tanto na adolescência, quanto em outras fases da vida 32,33. Desse modo, é relevante pontuar que o envolvimento em qualquer tipo de violência aumenta a chance de futuras vitimizações 7,11 . 
Figura 1 (continuação)

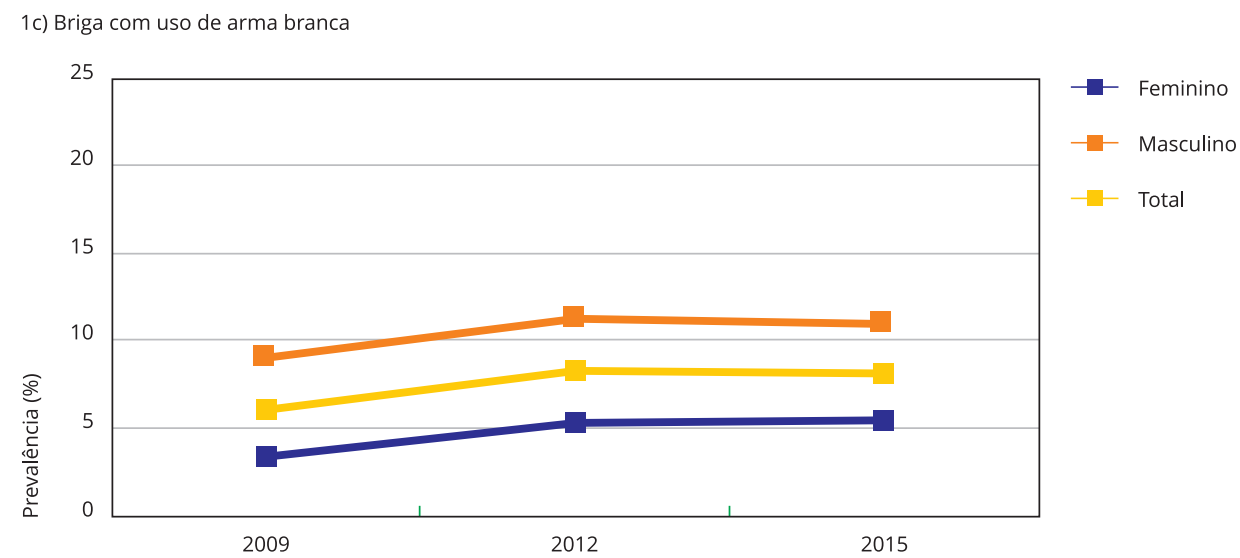

1d) Briga com uso de arma de fogo

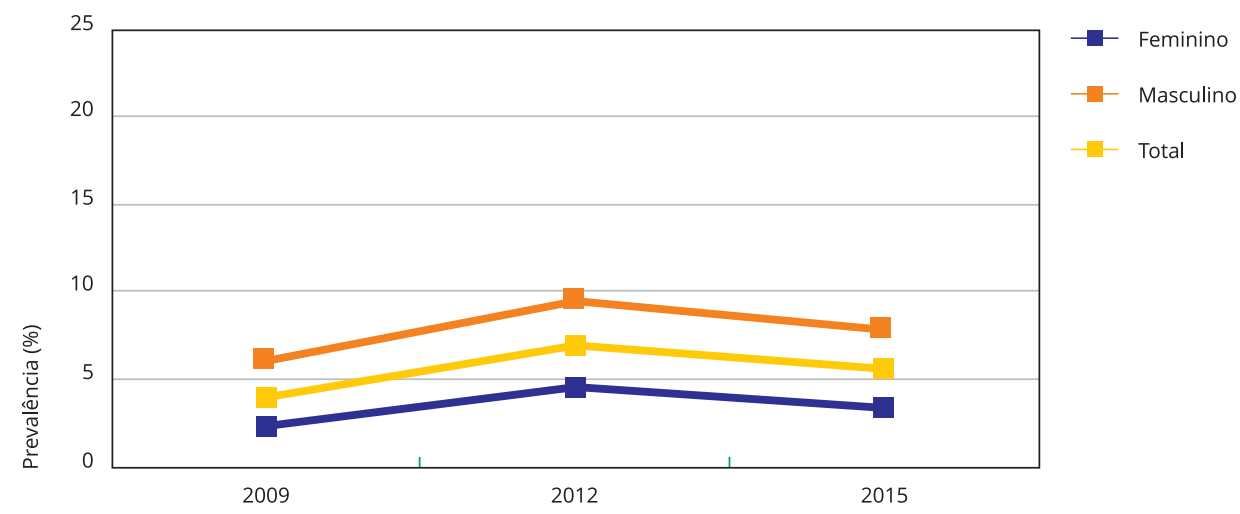

Nota: o modelo total foi ajustado para as variáveis sexo, escolaridade materna, idade, cor/raça, dependência administrativa da escola, uso de álcool, cigarro e drogas. Por outro lado, o modelo por sexo foi ajustado para todas as variáveis, exceto o sexo. Todos os modelos apresentaram tendência de aumento com valor de $p<0,001$.

Em relação ao bullying verbal, essa foi a forma de violência que mais vitimizou os adolescentes escolares analisados e foi um dos comportamentos com maior tendência de aumento. Uma possível explicação para as altas prevalências pode estar no fato de pais e professores reconhecerem esse tipo de violência como um comportamento normal durante o crescimento e o desenvolvimento das crianças e adolescentes. Além disso, destaca-se a ausência de políticas públicas claras de combate ao bullying 34 .

Aumento na prevalência de sofrer bullying também foi observado entre adolescentes venezuelanos no período de 2004 a 2008 35. Em contrapartida, tendência decrescente foi reportada entre adolescentes italianos no período de 2002 a 2010, sendo que em 2007 foram implementadas ações sistemáticas contra o bullying na Itália 36. Nos Estados Unidos, houve a implementação de programas antibullying no ambiente escolar, principalmente após ataques armados realizados nas escolas no final da década de 1990, e também foi observada a diminuição do bullying, assim como da vitimização por pares 14,37.

Nesse sentido, destacam-se as recentes ações de prevenção e combate ao bullying no Brasil, dentre elas a Lei no 13.185 38, sancionada em 2015, que instituiu o Programa de Combate à Intimidação Sistemática (bullying), visando à redução de atos violentos no ambiente escolar. Espera-se que, no 


\section{Figura 2}

Tendência de bullying verbal, violência doméstica, violência com arma branca e arma de fogo entre adolescentes escolares para o período de 2009 a 2015 nas capitais brasileiras.

2a) Bullying verbal

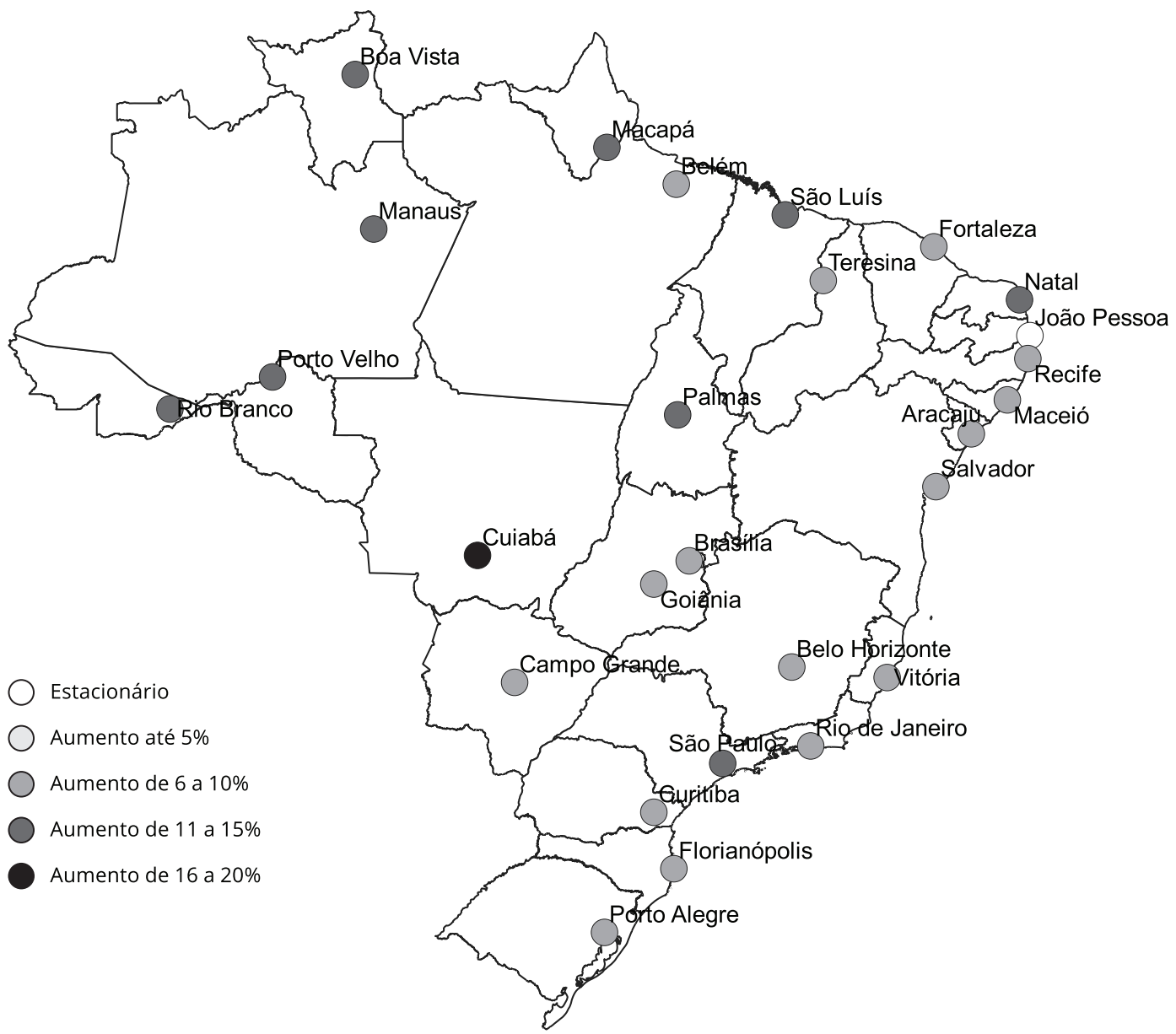

(continua)

decorrer do tempo, ocorra redução na frequência do bullying e que estudos futuros permitam avaliar a efetividade desse programa.

Entre adolescentes escolares do conjunto das capitais brasileiras, foram observadas tendências de aumento no envolvimento em brigas com utilização de armas de fogo e branca no período estudado (de 2009 a 2015). Uma explicação para esse aumento pode estar na presença de armas ilegais no Brasil 39, dado que, com a disponibilidade do armamento, há mais chances de envolvimento nesse tipo de violência, o que pode também afetar a quantidade de homicídios com uso de arma. Além do aumento no envolvimento em briga com uso de armas, também foi observado no país, na última década, crescimento nas taxas de homicídios com utilização de armas 3.

Em relação às prevalências, 8,2\% dos adolescentes relataram envolvimento em violência com uso de arma branca, e 5,6\% com uso de arma de fogo em 2015. Valores mais altos de vitimização direta, como envolvimento em briga com uso de arma de fogo ou branca, foram relatados por jovens nos 
2b) Violência doméstica

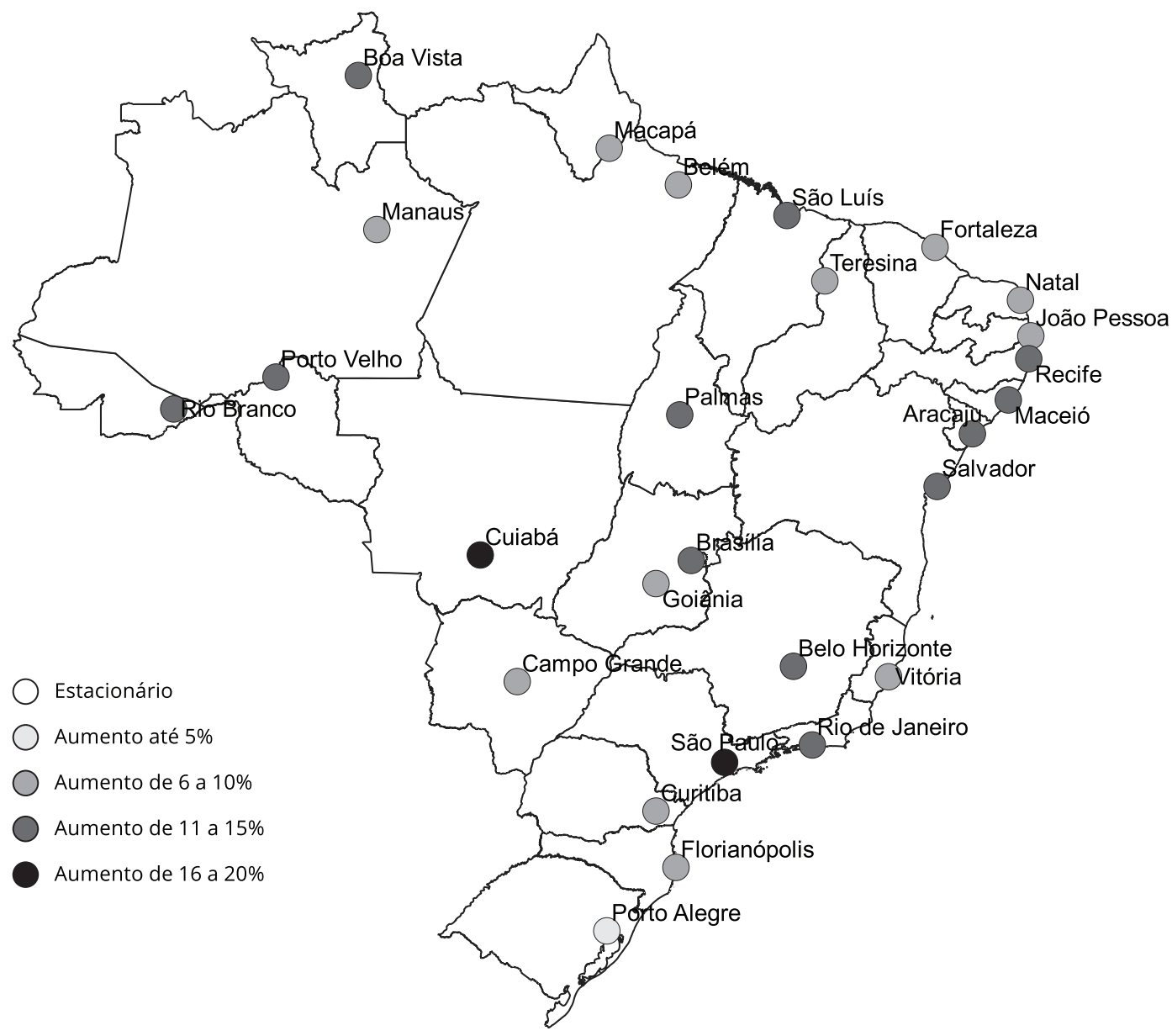

(continua)

Estados Unidos, onde cerca de 12,5\% haviam sido vítimas desse tipo de violência ao menos uma vez na vida 40 . Nota-se que o aumento no porte de arma de fogo foi relatado por adolescentes dos Estados Unidos, o que é um indicador proeminente de violência juvenil 41.

A menor prevalência entre adolescentes brasileiros quando comparados aos estadunidenses pode estar relacionada ao fato de, no Brasil, vigorar desde o início da década de 2000 o "Estatuto do Desarmamento", que restringe a comercialização de arma de fogo de acordo com alguns critérios, como idade mínima de 25 anos e declaração da necessidade do porte de tal armamento 42 . No entanto, mesmo com prevalências mais baixas do que as outras formas de violência analisadas, a violência com utilização de qualquer tipo de arma é preocupante, pois está associada a desfechos mais severos e com maior chance de letalidade 6 . No que se refere ao sexo das vítimas, para a maioria das violências, com exceção da violência doméstica, os meninos se envolveram com maior frequência em eventos violentos e também foram mais vitimizados, o que também é corroborado por outros estudos 11,40,43. Esse 


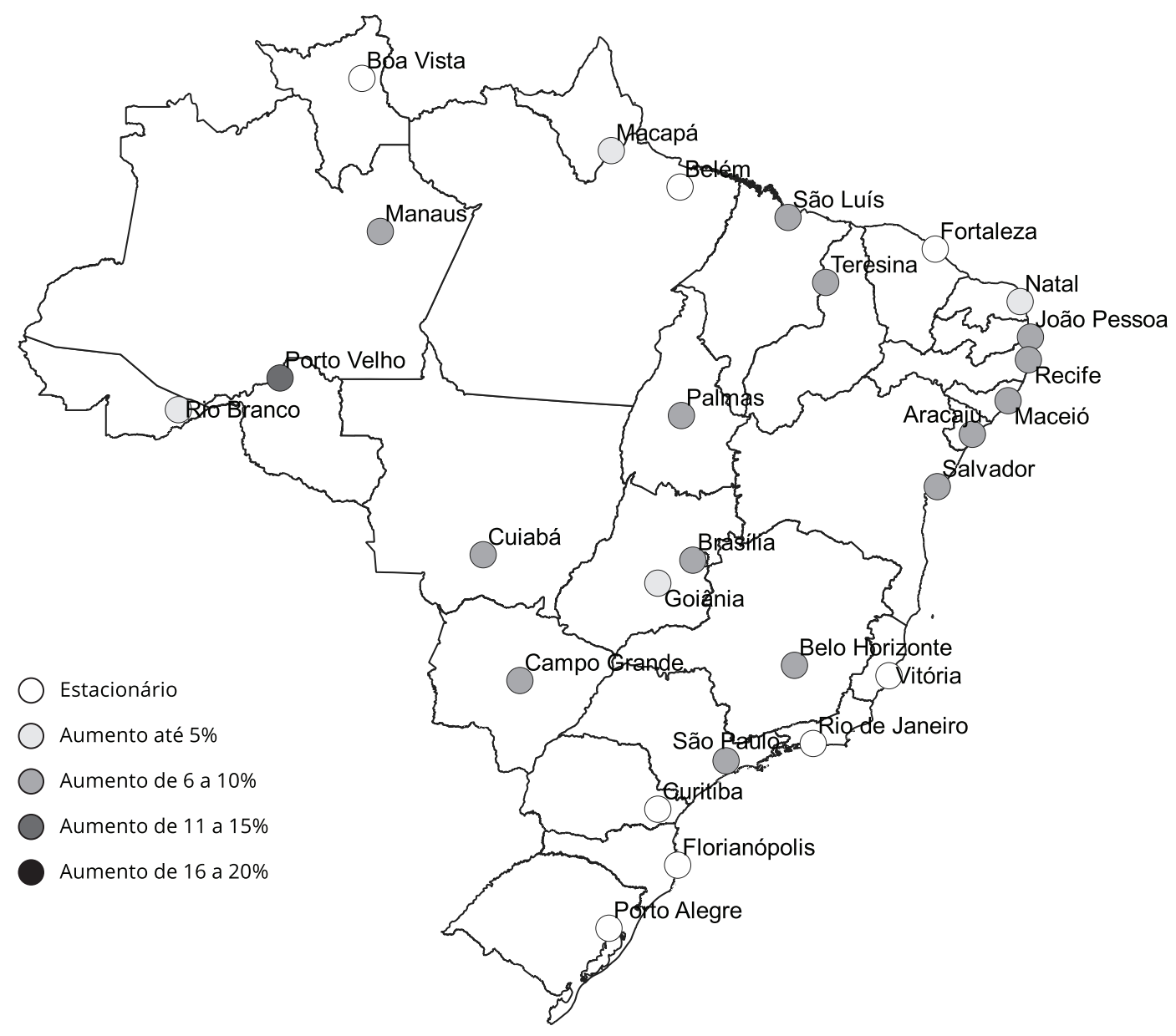

(continua)

fato pode ser explicado pela maior aceitação social de comportamentos violentos entre homens 44 .

Não foi possível identificar um padrão entre as capitais das cinco macrorregiões brasileiras. A Região Sul foi a única em que as capitais apresentaram tendências estacionárias para envolvimento em brigas com uso de armas de fogo e brancas. Esse fato pode ser explicado por ser uma região historicamente mais desenvolvida 45 e por possuir menor índice de desigualdade de renda 46 , que é um fator de risco para violência 47 . Entretanto, foram observadas prevalências mais acentuadas de envolvimento em briga com uso de arma de fogo e com uso de arma branca em capitais do Norte e do Nordeste. Essas regiões são menos desenvolvidas e possuem menores rendimentos médios mensais per capita que as demais 46 , o que corrobora a literatura que encontrou maiores taxas de violências em locais com baixas condições socioeconômicas 48 .

Está vigorando no Brasil, desde a década de 1990, o Estatuto da Criança e do Adolescente (ECA), que constitui um dos principais instrumentos para garantia de proteção contra todas as formas de violência que vitimizam crianças e adolescentes 49 . No entanto, a presença do ECA e de leis de proteção e combate à violência infanto-juvenil, por si só, não é suficiente para o enfrentamento do problema. 
2d) Briga com uso de arma de fogo

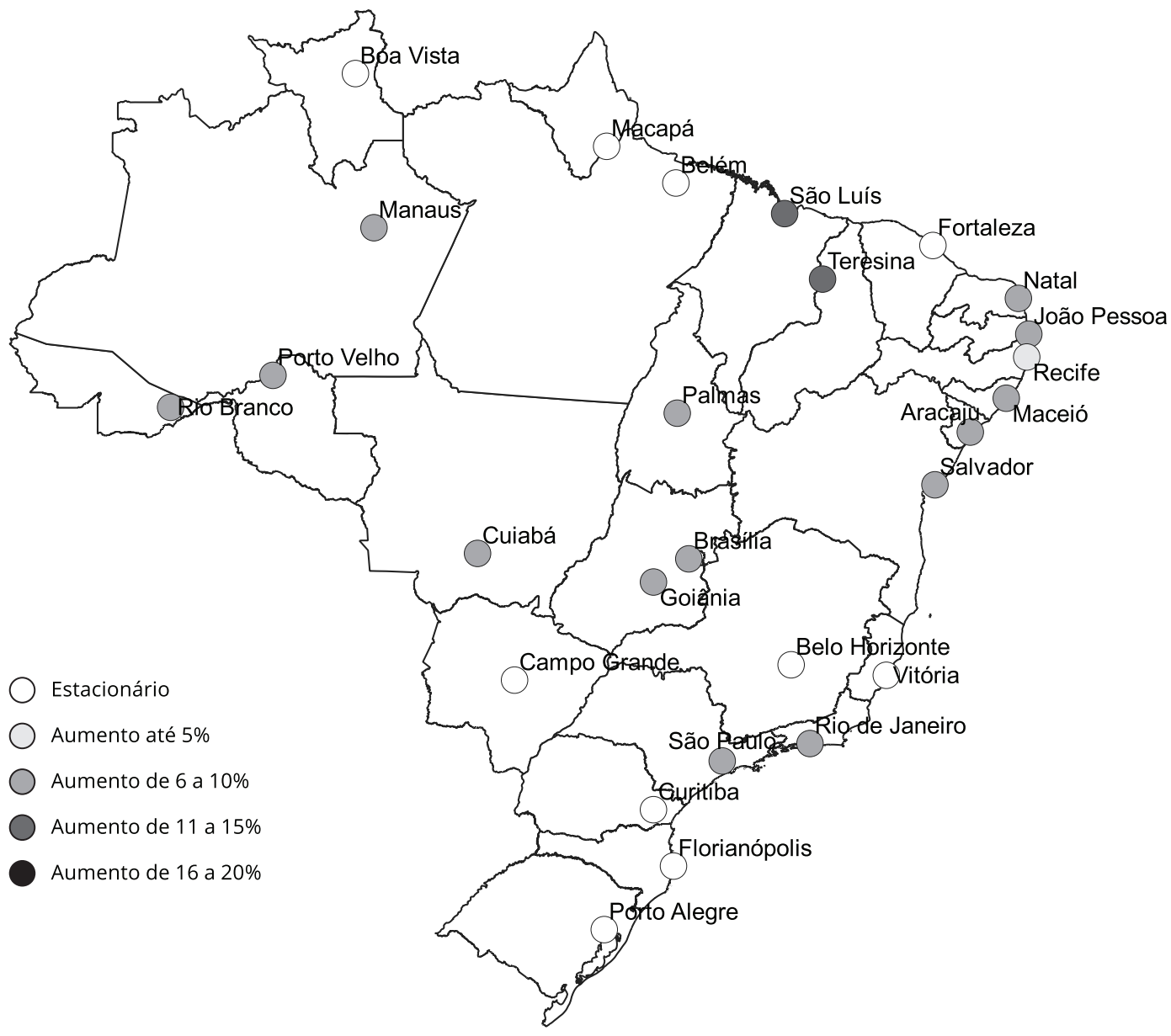

(continua)

Dessa forma, é necessário o envolvimento de outros setores da sociedade na adoção de medidas mais eficazes.

Além das legislações vigentes, vale destacar outras medidas para o enfrentamento da violência contra crianças e adolescentes. Dentre elas, devemos considerar a escola como um ambiente de intervenção, pois é um local de aprendizagem e de desenvolvimento de habilidades sociais e para a vida, que podem ser utilizadas para redução de comportamentos de risco para violência 50. Programa implementado em escolas da Colômbia, com foco na capacitação juvenil para resolução de problemas de forma não violenta, refletiu na redução da violência entre os escolares 51 . Ademais, no âmbito familiar, revisão sistemática mostrou que, principalmente nos Estados Unidos, programas direcionados para os pais adquirirem habilidades e conhecimentos em relação aos seus filhos e não utilizarem violência física como forma de correção resultaram em diminuição de comportamentos de risco, dentre eles violência familiar contra crianças e adolescentes 52 . Dessa forma, ações de enfrentamento e prevenção da violência contra crianças e adolescentes devem ser multissetoriais, envolvendo principalmente a escola e a família 47,50. 
A violência é um fenômeno global, presente em quase todas as sociedades 7 . No entanto, em populações de países de baixa e média renda, esse problema tende a ser mais acentuado 53,54, talvez por serem poucos os recursos investidos em educação e em programas de combate à violência. Por outro lado, foram observadas reduções nas taxas de violência em países de alta renda, como reflexo de estratégias desenvolvidas nos ambientes escolar e familiar, por exemplo 47.

Devemos considerar que esta pesquisa possui algumas limitações. Embora o estudo tenha analisado uma amostra representativa de escolares brasileiros, aqueles adolescentes que não estão matriculados em ensino regular não puderam ser representados, o que poderia gerar um viés de seleção. No entanto, como é alta a taxa de crianças e adolescentes que frequentam a escola, com 98,6\% de taxa de escolarização na faixa etária de 6 a 14 anos e de $85 \%$ dos 15 aos 17 anos 46, esse erro pode ter sido minimizado. Outro ponto faz referência à entrevista de adolescentes que frequentam a escola no dia da pesquisa. A literatura mostra que adolescentes que se envolvem em violência têm maiores taxas de absenteísmo escolar, e isso poderia influenciar as análises, subestimando a prevalência da violência 55. Além disso, as violências foram mensuradas a partir de perguntas únicas e referentes ao mês anterior à pesquisa, o que poderia ocasionar viés de informação. Outra limitação diz respeito ao fato de não sabermos se, nas violências com uso de armas de fogo ou branca, os escolares foram vítimas ou agressores. Apesar disso, não saber o papel do adolescente (se agressor ou vítima) não impossibilita o uso desse marcador para o planejamento de políticas públicas. Outro fator limitante é que os dados aqui apresentados são representativos dos escolares das capitais brasileiras, locais mais desenvolvidos e urbanizados que as cidades do interior. Por isso, podem não representar a realidade de escolares desses outros municípios.

Apesar das limitações, nossos resultados possuem pontos positivos. Nosso estudo utiliza dados de uma grande amostra com representatividade para as capitais brasileiras, com alta taxa de resposta nas três edições (superior a 81\%), o que reduz a possibilidade de viés de seleção. Outro ponto positivo é o uso de questionários autoaplicáveis, o que reduz possível viés de informação. Além disso, é o primeiro estudo que se propôs a avaliar a tendência temporal de quatro tipos de violência em escolares das capitais brasileiras. Por fim, possibilita a visualização da tendência da violência em cada uma das capitais e no Distrito Federal, em um país com realidades muito distintas e com grande extensão territorial.

Estudos futuros poderiam incluir adolescentes não matriculados em escolas, bem como aqueles que residem em outros municípios com características diferentes das capitais e dos grandes centros urbanos brasileiros. Além disso, a utilização de instrumentos validados e acurados para a identificação de violência juvenil poderia identificar maiores prevalências de violência. Também devemos destacar que estudos de tendência que avaliem dados de períodos futuros poderão melhor avaliar o impacto das políticas existentes, pois as políticas de enfrentamento ao bullying e à violência doméstica são recentes e talvez, com o passar dos anos, possam contribuir para a redução da violência.

O presente estudo possui importantes implicações, pois informa a magnitude, a evolução e os locais em que há maiores prevalências de quatro tipos de violência no Brasil, subsidiando o melhor direcionamento de ações de enfrentamento e de combate a esse problema. Conclui-se que, para o conjunto de capitais brasileiras, houve tendência de aumento para os quatro tipos de violência analisados, sem diferenças entre os sexos. A violência doméstica teve maior aumento, e as capitais do Norte e Nordeste apresentaram piores tendências para as formas mais graves de violência. Esses dados evidenciam que políticas nacionais de enfrentamento à violência direcionadas aos adolescentes em todas as capitais brasileiras devem contemplar os diferentes tipos de violência de forma conjunta, uma vez que grande parte dessa população pode estar exposta, direta ou indiretamente, a mais de um tipo de violência. 


\section{Colaboradores}

A. N. Silva e C. M. Azeredo atuaram nas etapas de concepção do estudo, análise de dados, redação final do artigo e aprovação da versão final do manuscrito. E. S. Marques e M. F. T. Peres contribuíram com a redação e revisão do manuscrito e aprovaram a versão final do texto.

\section{Informações adicionais}

ORCID: Aline Natália Silva (0000-0002-18113703); Emanuele Souza Marques (0000-00028633-7290); Maria Fernanda Tourinho Peres (00000002-7049-905X); Catarina Machado Azeredo (0000-0002-6189-4429).

\section{Agradecimentos}

Gostaríamos de agradecer a Arlei Teodoro de Queiroz, pela ajuda na elaboração dos mapas. À Coordenação de Aperfeiçoamento de Pessoal de Nível Superior (Capes), pela concessão de bolsa de pósgraduação para a mestranda Aline Natália Silva, e ao apoio financeiro do Conselho Nacional de Desenvolvimento Científico e Tecnológico (CNPq; $\left.n^{\circ} 404905 / 2016-1\right)$, concedido a Catarina Machado Azeredo. Agrademos também ao Programa de Pós-graduação em Saúde Coletiva do Instituto de Medicina Social da Universidade do Estado do Rio de Janeiro, que forneceu auxílio financeiro para a publicação do artigo.

\section{Referências}

1. Organização Mundial da Saúde. Relatório mundial sobre a prevenção da violência: 2014. Genebra: Organização Mundial da Saúde; 2014.

2. United Nations Children's Fund. A familiar face: violence in the lives of children and adolescents. New York: United Nations Children's Fund; 2017.

3. Instituto de Pesquisa Econômica Aplicada. Atlas da violência. Rio de Janeiro: Instituto de Pesquisa Econômica Aplicada; 2018.

4. Patton GC, Sawyer SM, Santelli JS, Ross DA, Afifi R, Allen NB, et al. Our future: a Lancet commission on adolescent health and wellbeing. Lancet 2016; 387:2423-78.

5. Copeland WE, Wolke D, Angold A, Costello EJ. Adult psychiatric outcomes of bullying and being bullied by peers in childhood and adolescence. JAMA Psychiatry 2013; 70:419-26.

6. David-Ferdon C, Simon TR, Spivak H, Gorman-Smith D, Savannah SB, Listenbee RL. CDC Grand Rounds: preventing youth violence. MMWR Morb Mortal Wkly Rep 2015; 64:171-4.

7. Krug EG, Dahlberg LL, Mercy JA, Zwi AB, Lozano R. World report on violence and health. Geneva: World Health Organization; 2002.

8. Park S, Lee Y, Jang H, Jo M. Violence victimization in Korean adolescents: risk factors and psychological problems. Int J Environ Res Public Health 2017; 14:E541.

9. Pool AC, Patterson F, Luna IY, Hohl B, Bauer KW. Ten-year secular trends in youth violence: results from the Philadelphia Youth Risk Behavior Survey 2003-2013. J Sch Health 2017; 87:244-52.

10. Shavers CA. Exposures to violence and trauma among children and adolescents in our global society. Psychology 2013; 4:133-5.

11. Peres MFT, Azeredo CM, de Rezende LFM, Zucchi EM, Franca-Junior I, Luiz OC, et al. Personal, relational and school factors associated with involvement in fights with weapons among school-age youth in Brazil: a multilevel ecological approach. Int J Public Health 2018; 63:957-65.

12. Salas-Wright CP, Nelson EJ, Vaughn MG, Reingle Gonzalez JM, Córdova D. Trends in fighting and violence among adolescents in the United States, 2002-2014. Am J Public Health 2017; 107:977-82.

13. Finkelhor D, Shattuck A, Turner HA, Hamby SL. Trends in children's exposure to violence, 2003 to 2011. JAMA Pediatr 2014; 168:540-6.

14. Finkelhor D, Turner H, Ormrod R, Hamby SL. Trends in childhood violence and abuse exposure: evidence from 2 national surveys. Arch Pediatr Adolesc Med 2010; 164:238-42.

15. Djerboua M, Chen BE, Davison C. Trends and demographic characteristics of physical fighting and fighting-related injuries among $\mathrm{Ca}$ nadian youth, 1993-2010. Can J Public Health 2016; 107:231-8. 
16. Villela LCM, Moraes SA, Suzuki CS, Freitas ICM. Tendência da mortalidade por homicídios em Belo Horizonte e região metropolitana: 1980-2005. Rev Saúde Pública 2010; 44:486-95.

17. Peres MFT, Nivette A. Social disorganization and homicide mortality rate trajectories in Brazil between 1991 and 2010. Soc Sci Med 2017; 190:92-100.

18. Mello FCM, Malta DC, Santos MG, Silva MMA, Silva MAI. Evolução do relato de sofrer bullying entre escolares brasileiros: Pesquisa Nacional de Saúde do Escolar - 2009 a 2015. Rev Bras Epidemiol 2018; 21 Suppl 1:e180015.

19. Pinto IV, Barufaldi LA, Campos MO, Malta DC, Souto RMCV, Freitas MG, et al. Tendências de situações de violência vivenciadas por adolescentes brasileiros: Pesquisa Nacional de Saúde do Escolar 2009, 2012 e 2015. Rev Bras Epidemiol 2018; 21 Suppl 1:e180014.

20. Instituto Brasileiro de Geografia e Estatística. Pesquisa Nacional de Saúde do Escolar: 2009. Rio de Janeiro: Instituto Brasileiro de Geografia e Estatística; 2009.

21. Instituto Brasileiro de Geografia e Estatística. Pesquisa Nacional de Saúde do Escolar: 2012. Rio de Janeiro: Instituto Brasileiro de Geografia e Estatística; 2013.

22. Instituto Brasileiro de Geografia e Estatística. Pesquisa Nacional de Saúde do Escolar: 2015. Rio de Janeiro: Instituto Brasileiro de Geografia e Estatística; 2016.

23. Oliveira MM, Campos MO, Andreazzi MAR, Malta DC, Oliveira MM, Campos MO, et al. Características da Pesquisa Nacional de Saúde do Escolar - PeNSE. Epidemiol Serv Saúde 2017; 26:605-16.

24. Olweus, D. Bullying at school: what we know and what we can do. Malden: Blackwell Publishing: 1993.

25. Royston P, White I. Multiple Imputation by Chained Equations (MICE): implementation in Stata. J Stat Softw 2011; 45:1-20.

26. Brasil. Lei no 13.010, de 26 de junho de 2014. Altera a Lei no 8.069, de 13 de julho de 1990 (Estatuto da Criança e do Adolescente), para estabelecer o direito da criança e do adolescente de serem educados e cuidados sem o uso de castigos físicos ou de tratamento cruel ou degradante, e altera a Lei no 9.394, de 20 de dezembro de 1996. Diário Oficial da União 2014; 3 jul.

27. Martins CBG, Jorge MHPM. A violência contra crianças e adolescentes: características epidemiológicas dos casos notificados aos Conselhos Tutelares e programas de atendimento em município do Sul do Brasil, 2002 e 2006. Epidemiol Serv Saúde 2009; 18:315-34.

28. Moreira MIC, Sousa SMG. Violência intrafamiliar contra crianças e adolescentes: do espaço privado à cena pública. O Social em Questão 2012; 15:13-26.

29. Barros MMAC, Rocha GOR. Crianças e adolescentes e o direito de não violência sexual: conceitos, políticas públicas e legislação. InterMeio: Revista do Programa de Pós-Graduação em Educação 2015; 21:67-83.
30. Gilbert R, Fluke J, O’Donnell M, Gonzalez-Izquierdo A, Brownell M, Gulliver P, et al. Child maltreatment: variation in trends and policies in six developed countries. Lancet 2012; 379:758-72.

31. World Health Organization. Violence prevencion: the evidence. Geneva: World Health Organization; 2010.

32. Braga T, Gonçalves LC, Basto-Pereira M, Maia $\hat{A}$. Unraveling the link between maltreatment and juvenile antisocial behavior: a meta-analysis of prospective longitudinal studies. Aggress Violent Behav 2017; 33:37-50.

33. Cecconello AM, De Antoni C, Koller SH. Práticas educativas, estilos parentais e abuso físico no contexto familiar. Psicol Estud 2003; 8:4554.

34. United Nations Educational, Scientific and Cultural Organization. School violence and bullying global status report. Paris: United $\mathrm{Na}$ tions Educational, Scientific and Cultural Organization; 2017.

35. Granero R, Poni ES, Escobar-Poni BC, Escobar J. Trends of violence among 7 th, 8 th and 9th grade students in the state of Lara, Venezuela: The Global School Health Survey 2004 and 2008. Arch Public Health 2011; 69:7.

36. Vieno A, Lenzi M, Gini G, Pozzoli T, Cavallo F, Santinello M. Time trends in bullying behavior in Italy. J Sch Health 2015; 85:441-5.

37. Ferguson CJ, Miguel CS, Kilburn JC, Sanchez P. The effectiveness of school-based anti-bullying programs: a meta-analytic review. Crim Justice Rev 2007; 32:401-14.

38. Brasil. Lei no 13.185 , de 6 de novembro de 2015. Institui o Programa de Combate à Intimidação Sistemática (Bullying). Diário Oficial da União 2015; 9 nov.

39. Scorzafave LG, Soares MK, Dorigan TA. Vale a pena pagar para desarmar? Uma avaliação do impacto da campanha de entrega voluntária de armas sobre as mortes com armas de fogo. Estudos Econômicos 2015; 45:475-97.

40. Mitchell KJ, Hamby SL, Turner HA, Shattuck A, Jones LM. Weapon involvement in the victimization of children. Pediatrics 2015; 136:10-7.

41. Perlus JG, Brooks-Russell A, Wang J, Iannotti RJ. Trends in bullying, physical fighting, and weapon carrying among 6th- through 10thgrade students from 1998 to 2010: findings from a national study. Am J Public Health 2014; 104:1100-6.

42. Brasil. Decreto no 5.123, de 1o de julho de 2004. Regulamenta a Lei no 10.826 , de 22 de dezembro de 2003, que dispõe sobre registro, posse e comercialização de armas de fogo e munição, sobre o Sistema Nacional de Armas - SINARM e define crimes. Diário Oficial da União 2004; 2 jul.

43. Azeredo CM, Levy RB, Araya R, Menezes PR. Individual and contextual factors associated with verbal bullying among Brazilian adolescents. BMC Pediatr 2015; 15:49-56. 
44. Baxendale S, Cross D, Johnston R. A review of the evidence on the relationship between gender and adolescents' involvement in violent behavior. Aggress Violent Behav 2012; 17:297310.

45. Reichenheim ME, de Souza ER, Moraes CL, de Mello Jorge MHP, da Silva CMFP, de Souza Minayo MC. Violence and injuries in Brazil: the effect, progress made, and challenges ahead. Lancet 2011; 377:1962-75.

46. Instituto Brasileiro de Geografia e Estatística. Pesquisa Nacional por Amostra de Domícílio: síntese de indicadores. Rio de Janeiro: Instituto Brasileiro de Geografia e Estatística; 2016.

47. Organização Mundial da Saúde. Prevenindo a violência juvenil: um panorama das evidências. Genebra: Organização Mundial da Saúde; 2015.

48. Otwombe KN, Dietrich J, Sikkema KJ, Coetzee J, Hopkins KL, Laher F, et al. Exposure to and experiences of violence among adolescents in lower socio-economic groups in Johannesburg, South Africa. BMC Public Health 2015, 15:450.

49. Brasil. Lei no 8.069, de 13 de julho de 1990. Dispõe sobre o Estatuto da Criança e do Adolescente e dá outras providências. Diário Oficial da União 1990; 27 set.
50. World Health Organization. INSPIRE: seven strategies for ending violence against children. Geneva: World Health Organization; 2016.

51. Chaux E. Aulas en paz: a multicomponent program for the promotion of peaceful relationships and citizenship competencies. Conflict Resolution Quarterly 2007; 25:79-86.

52. Peacock S, Konrad S, Watson E, Nickel D, Muhajarine N. Effectiveness of home visiting programs on child outcomes: a systematic review. BMC Public Health 2013; 13:17.

53. Naghavi M, Abajobir AA, Abbafati C, Abbas KM, Abd-Allah F, Abera SF, et al. Global, regional, and national age-sex specific mortality for 264 causes of death, 1980-2016: a systematic analysis for the Global Burden of Disease Study 2016. Lancet 2017; 390:1151-210.

54. Wolf A, Gray R, Fazel S. Violence as a public health problem: an ecological study of 169 countries. Soc Sci Med 2014; 104:220-7.

55. Melo ACM, Garcia LP. Involvement of school students in fights with weapons: prevalence and associated factors in Brazil. BMC Public Health 2016; 16:1008. 


\section{Abstract}

This study analyzes time trends in verbal bullying, domestic violence, and involvement in fights with firearms among adolescents in Brazilian state capitals from 2009 to 2015. The study of trends uses data from the Brazilian National Survey of School Health (PeNSE) in 2009, 2012, and 2015 among ninth-graders enrolled in public and private schools in the country's 26 state capitals and the Federal District. The analysis focused on domestic violence, verbal bullying, and involvement in fights with cold steel weapons and firearms in the 30 days prior to the interview. Logistic regression was performed, adjusted for sociodemographic and behavioral characteristics, with analysis of each type of violence in Brazil and in the capital cities, according to sex. The trends were spatialized. For the capital cities as a whole, from 2009 to 2015 there was an increase of 12\% (95\% CI: 1.111.14) in domestic violence, $10 \%$ (95\% CI: 1.08-1.11) in verbal bullying, and 7\% (95\%CI: 1.05-1.09) and 7\% (95\% CI: 1.05-1.08) in involvement in fights with firearms and cold steel weapons, respectively. Domestic violence increased in all the capital cities. In 96.3\%, 70.4\%, and $62.9 \%$ of the capital cities, respectively, there were increases in verbal bullying and involvement in fights with cold steel weapons and firearms, while there were stationary trends in the other capital cities. There was an upward trend in these forms of violence in the majority of the capital cities, evidencing the need to implement public policies to mitigation the different types of violence among adolescents.

Time Series Studies; Violence; Adolescent

\section{Resumen}

El objetivo del estudio fue analizar la tendencia temporal de bullying verbal, violencia doméstica e implicación en peleas con armas entre adolescentes en las capitales brasileñas entre 2009 y 2015. Se trata de un estudio de tendencia, usando datos de la Encuesta Nacional de Salud del Escolar (PeNSE) realizadas en 2009, 2012 y 2015, con escolares del 9o año de enseñanza fundamental, en escuelas públicas y privadas de las 26 capitales del país y Distrito Federal. Se analizó la ocurrencia de violencia doméstica, de bullying verbal, así como la implicación en peleas con uso de armas blancas $y$ de fuego durante los 30 días anteriores a la entrevista. Se realizó una regresión logística ajustada para características sociodemográficas y comportamentales, con análisis de cada tipo de violencia en Brasil y en las capitales, según el sexo. Las tendencias fueron espacializadas. Para el conjunto de capitales hubo un aumento, entre 2009 y 2015, de un 12\% (IC95\%: 1,11-1,14) para violencia doméstica, de un 10\% (IC95\%: 1,08-1,11) para bullying verbal, de un 7\% (IC95\%: 1,05-1,09) y 7\% (IC95\%: 1,05-1,08) para implicación en peleas con armas de fuego y blanca, respectivamente. En todas las capitales hubo un aumento de la violencia doméstica. En un 96,3\%, 70,4\% y 62,9\% de las capitales se observó el aumento de bullying verbal, implicación en peleas con armas blancas y de fuego, respectivamente. En las demás capitales, se observó una tendencia estacionaria. Se identificó una tendencia de aumento de la violencia en la mayoría de las capitales, mostrando la necesidad de implementación de políticas públicas que contribuyan a la minimización de los diferentes tipos de violencia entre los adolescentes.

Estudios de Series Temporales; Violencia; Adolescente
Recebido em 16/Out/2018

Versão final reapresentada em 14/Mai/2019 Aprovado em 24/Mai/2019 